\title{
Two field BPS solutions for generalized Lorentz breaking models
}

\author{
A. de Souza Dutra, ${ }^{1,2, *}$ M. Hott, ${ }^{2, \dagger}$ and F. A. Barone ${ }^{2, \ddagger}$ \\ ${ }^{1}$ Abdus Salam ICTP, Strada Costiera 11, Trieste, I-34100 Italy \\ ${ }^{2}$ UNESP-Campus de Guaratinguetá-DFQ ${ }^{\S}$, Departamento de Física e Química, 12516-410 Guaratinguetá SP, Brasil
}

(Received 21 July 2006; published 30 October 2006)

\begin{abstract}
In this work we present nonlinear models in two-dimensional space-time of two interacting scalar fields in the Lorentz and $C P T$ violating scenarios. We discuss the soliton solutions for these models as well as the question of stability for them. This is done by generalizing a model recently published by Barreto and collaborators and also by getting new solutions for the model introduced by them.
\end{abstract}

DOI: 10.1103/PhysRevD.74.085030

PACS numbers: $11.15 . \mathrm{Kc}, 11.27 .+\mathrm{d}$

Some years ago, Carrol et al. [1] started to analyze the problem of Lorentz breaking signature in field theoretical models. By now, there are a great number of works discussing a kind of symmetry breaking in many different physically interesting contexts. For instance, in [2] some impact over the standard model of this kind of symmetry breaking was discussed. Azatov et al. [3], in a recent work, have analyzed the spontaneous breaking of the fourdimensional Lorentz invariance of the QED through a nonlinear vector potential constraint, Bezerra et al. [4] have shown that a space-time with torsion interacting with a Maxwell field by means of a Chern-Simons-like term can explain the optical activity in the synchrotron radiation emitted by cosmological distant radio sources, Lehnert et al. [5] have verified the consequences over the Cerenkov effect of a Lorentz-violating vacuum, and Bluhm [6] has made an estimative analysis of the Lorentz and $C P T$ bounds attainable in Penning-trap experiments. In fact, over the last years a considerable effort has been drawn into this direction by many groups and in a variety of physical applications. On the other hand, the presence of topological solutions of nonlinear models is a matter of large interest and possible applications [7-9]. On the other hand, a natural place to apply these ideas is that of condensed matter nonrelativistic ground, where the break of isotropy and homogeneity emerges quite naturally, due the material structure.

As a consequence of the above arguments, it is natural to look for topological structures in $C P T$ breaking scenarios. In fact, in a very recent work in this journal, Barreto et al. [10] have introduced an approach capable of getting kinks in $C P T$ violating scenarios.

Here we are going to discuss a generalization of the work of Ref. [10], both by obtaining more general solutions for the models considered on that work and by

\footnotetext{
*Electronic address: dutra@feg.unesp.br

${ }^{\dagger}$ Electronic address: hott@feg.unesp.br

Electronic address: fbarone@feg.unesp.br

${ }^{\S}$ Permanent institution
}

generalizing Lorentz breaking Lagrangian densities. Particularly we obtain solutions which were absent in Ref. [10]. For this last accomplishment, we use a method recently introduced by one of us [11].

Models with Lorentz symmetry breaking terms usually lead to nonlinear differential equations, and one of the problems appearing as a consequence of this nonlinearity is that, in general, we lose the capability of getting the complete solutions. Here we extend an approach exposed in Ref. [11] which shows that for some two-field systems in $1+1$ dimensions, whose second-order differential equations can be reduced to the solution of corresponding firstorder equations (the so-called Bolgomol'nyi-PrasadSommerfield (BPS) topological solitons [12]), one can obtain a differential equation relating the two coupled fields which, once solved, lead to the general orbit connecting the vacua of the model. In fact, the "trial and error" methods historically arose as a consequence of the intrinsic difficulty of getting general methods of solution for nonlinear differential equations. About two decades ago, Rajaraman [7] introduced an approach of this nature for the treatment of coupled relativistic scalar field theories in $1+1$ dimensions. His procedure was model independent and could be used for the search of solutions in arbitrary coupled scalar models in $1+1$ dimensions. However, the method is limited in terms of the generality of the solutions obtained and is convenient and profitable only for some particular, but important, cases [13]. Some years later, Bazeia and collaborators [14] applied the approach developed by Rajaraman to special cases where the solution of the nonlinear second-order differential equations are equivalent to the solution of corresponding firstorder nonlinear coupled differential equations. In this work we are going to present a procedure which is absolutely general when applied to Lorentz and $C P T$ breaking systems, like those obtained from an extension of the ones described in [11] applied to no Lorentz symmetry breaking versions appearing in [14-19]. Furthermore, we also show that many of these systems can be mapped into a first-order linear differential equation and, as a consequence, can be 
solved in order to get the general solution of the system. After that, we trace some comments about the consequences coming from these general solutions.

\section{BPS NONLINEAR LORENTZ AND CPT SCENARIOS}

The two-field model we shall study in $1+1$ dimensions is described by the Lagrangian density

$$
\begin{aligned}
\mathcal{L}= & \frac{1}{2}\left(\partial_{\mu} \phi\right)^{2}+\frac{1}{2}\left(\partial_{\mu} \chi\right)^{2}-f^{\mu}(\phi, \chi) \partial_{\mu} \chi \\
& -g^{\nu}(\phi, \chi) \partial_{\nu} \phi-V(\phi, \chi),
\end{aligned}
$$

where $\mu=0,1, f^{\mu}(\phi, \chi)$, and $g^{\nu}(\phi, \chi)$ are vector functions with a prescribed functional dependence on the dynamical fields $\phi$ and $\chi$, and $V(\phi, \chi)$ is a potential term.

Note that we can recover some usual Lorentz symmetry breaking models from (1) by choosing appropriately the vectors $f^{\mu}$ and $g^{\mu}$. In particular, if $f_{1}=s_{2} \phi$ and $g_{1}=$ $s_{1} \chi$, one recovers the model introduced very recently by Barreto and collaborators [10]. In fact, the first example we work out here is precisely this one, which we are going to show possesses an entire topological sector not considered in the work of Ref. [10].

If the potential $V(\phi, \chi)$ can be written in such a way that

$$
\begin{aligned}
V(\phi, \chi)= & \frac{1}{2}\left(\frac{d W(\phi, \chi)}{d \phi}-g_{1}(\phi, \chi)\right)^{2} \\
& +\frac{1}{2}\left(\frac{d W(\phi, \chi)}{d \chi}-f_{1}(\phi, \chi)\right)^{2},
\end{aligned}
$$

with $W(\phi, \chi)$ being any function of $\phi$ and $\chi$, the energy density of the BPS states becomes

$$
\begin{aligned}
\mathcal{E}_{\mathrm{BPS}}= & \frac{1}{2}\left(\frac{d \phi}{d x}-\frac{d W(\phi, \chi)}{d \phi}+g_{1}(\phi, \chi)\right)^{2} \\
& +\frac{1}{2}\left(\frac{d \chi}{d x}-\frac{d W(\phi, \chi)}{d \chi}+f_{1}(\phi, \chi)\right)^{2}+\frac{d W}{d x},
\end{aligned}
$$

with $d W / d x=W_{\phi} \phi^{\prime}+W_{\chi} \chi^{\prime}$, where we have defined $W_{\phi} \equiv \frac{\partial W}{\partial \phi}, W_{\chi} \equiv \frac{\partial W}{\partial \chi}$ and the prime stands for space derivative.

From Eq. (3), we can see that the solutions of minimal energy are obtained from the following two coupled firstorder equations

$$
\begin{aligned}
\phi^{\prime} & =W_{\phi}(\phi, \chi)-g_{1}(\phi, \chi), \\
\chi^{\prime} & =W_{\chi}(\phi, \chi)-f_{1}(\phi, \chi) .
\end{aligned}
$$

Finally the BPS energy is written, as usual, by

$$
E_{\mathrm{BPS}}=\left|W\left(\phi_{j}, \chi_{j}\right)-W\left(\phi_{i}, \chi_{i}\right)\right|,
$$

where $\phi_{i}$ and $\chi_{i}$ mean the $i$ th vacuum states of the model. Here, it is important to remark that the BPS solutions settle into vacuum states asymptotically. In other words, the vacuum states act as implicit boundary conditions of the BPS equations.
It is interesting to notice that in the first-order equations of motion (4) and in the energy density (3) only the space components of the functional vectors $f_{\mu}$ and $g_{\mu}, f_{1}$ and $g_{1}$, respectively, are present.

From now on, in order to solve Eqs. (4), let us consider models for which we can write $\phi$ as a function of $\chi$, that is, $\phi(\chi)$. In this situation, instead of applying the usual trialorbit approach [14-19], we note that it is possible to write the following equation

$$
\frac{d \phi}{W_{\phi}-g_{1}}=d x=\frac{d \chi}{W_{\chi}-f_{1}},
$$

where the differential element $d x$ is a kind of invariant. In these cases one is lead to

$$
\frac{d \phi}{d \chi}=\frac{W_{\phi}-g_{1}}{W_{\chi}-f_{1}} .
$$

Equation (7) is the generalization of the one studied in [11] to the case of nonlinear Lorentz and $C P T$ breaking scenarios. It is, in general, a nonlinear differential equation relating the scalar fields of the model. If one is able to solve it completely for a given model, the function $\phi(\chi)$ can be used to eliminate one of the fields, so rendering Eqs. (4) uncoupled and equivalent to a single one. Finally, this uncoupled first-order nonlinear equation can be solved in general, even if numerically.

We have found this method simpler than the method of the orbits broadly and successfully applied to study the mapping of the soliton solutions and defect structures in problems involving the interaction of two scalar fields. Despite being simpler, the method applied here furnishes not only the same orbits as those obtained by using the method of the orbits appearing in the Refs. [14-19], but also some new ones, as can be seen through a comparison with Ref. [11]. In the example worked out below one can verify that, this time, the mapping constructed here furnishes the very same orbits obtained in Ref. [10]. Notwithstanding, we are able to find new solitonic configurations, not observed by Barreto and collaborators.

\section{THE EXAMPLE OF LINEAR LORENTZ AND CPT BREAKING}

In this section we consider the particular model introduced in the work of Barreto et al [10] in order to apply the method discussed in the previous section. In fact, we show in this example that Eq. (7) can be mapped into a linear differential equation, from which it is possible to obtain the general solutions for the soliton fields. In the case on the screen, the superpotential [10] is written as

$$
W(\phi, \chi)=\phi-\frac{1}{3} \phi^{3}-r \phi \chi^{2},
$$

and the Lorentz symmetry breaking terms in the Lagrangian density (1) are chosen to be given by $f_{1}(\phi, \xi)=s_{2} \phi$ and $g_{1}(\phi, \xi)=s_{1} \chi$, such that Eq. (7) is 
rewritten as

$$
\frac{d \phi}{d \chi}=\frac{\left(\phi^{2}-1\right)+r \chi^{2}+s_{1} \chi}{2 r \phi \chi+s_{2} \phi},
$$

where $s_{1}$ and $s_{2}$ are constants.

At this point one can verify that, performing the transformations

$$
\chi=\zeta-\frac{s_{2}}{2 r}
$$

and

$$
\phi^{2}=\rho+1+\frac{s_{2}}{4 r}\left(2 s_{1}-s_{2}\right),
$$

Eq. (9) becomes

$$
\frac{d \rho}{d \zeta}-\frac{\rho}{r \zeta}=\zeta-\frac{b}{r}
$$

which is a typical inhomogeneous linear differential equation [11]. The general solutions for the orbit equation are then easily obtained, giving

$$
\begin{aligned}
& \phi^{2}-1=c_{0} \zeta^{1 / r}+\frac{r}{2 r-1} \zeta^{2}-\frac{b}{r-1} \zeta+k \\
& \text { for } r \neq 1 \text { and } r \neq \frac{1}{2}, \\
& \phi^{2}-1=-b \zeta \ln (\zeta)+c_{1} \zeta+\zeta^{2}+k \text { for } r=1,
\end{aligned}
$$

and

$$
\phi^{2}-1=\zeta^{2} \ln (\zeta)+b \zeta+c_{2} \zeta^{2}+k \text { for } r=\frac{1}{2},
$$

where $k \equiv \frac{s_{2}}{4 r}\left(2 s_{1}-s_{2}\right), b \equiv s_{2}-s_{1}$ and $c_{0}, c_{1}$, and $c_{2}$ are arbitrary integration constants.

In general it is not possible to solve $\chi$ in terms of $\phi$ from the above solutions, but the contrary is always granted. Here, with the aid of (8) and (10), we shall substitute the expressions of $\phi(\chi)$ obtained from (13)-(15) in the second Eq. (4), obtaining respectively:

$$
\begin{aligned}
& \frac{d \zeta}{d x}= \pm 2 r \zeta \sqrt{1+c_{0} \zeta^{1 / r}+\frac{r}{2 r-1} \zeta^{2}-\frac{b}{r-1} \zeta+k} \\
& \quad \text { for } r \neq 1, \quad r \neq \frac{1}{2},
\end{aligned}
$$

$$
\begin{array}{ll}
\frac{d \zeta}{d x}= \pm 2 r \zeta \sqrt{1-b \zeta \ln (\zeta)+c_{1} \zeta+\zeta^{2}+k} & \text { for } r=1, \\
\frac{d \zeta}{d x}= \pm 2 r \zeta \sqrt{1+\zeta^{2} \ln (\zeta)+b \zeta+c_{2} \zeta^{2}+k} \quad \text { for } r=\frac{1}{2} .
\end{array}
$$

Barreto and collaborators [10] have limited themselves to the orbits in which $r \neq 1$ and $r \neq 1 / 2$ and the arbitrary constant $c_{0}$ equals zero or infinity. In the particular case with $c_{0}=0$ they have found a lumplike profile for the field $\chi(x)$ and a kinklike profile for the field $\phi(x)$. By integrating
Eq. (16) and substituting its solutions into Eq. (10) we get the following solutions for the field $\chi(x)$

$$
\chi_{ \pm}^{A}(x)=\frac{4 \sqrt[3]{A} e^{\mp 2 \sqrt{A} r\left(x-x_{0}\right)}}{\left(\sqrt{A} e^{\mp 2 \sqrt{A} r\left(x-x_{0}\right)}+C\right)^{2}-4 A B}-\frac{b}{2 r},
$$

where $x_{0}$ is a constant of integration, $A=1-b^{2} / 4 r, B=$ $r /(2 r-1), C=b /(r-1)$ and we have taken $s_{1}=0$. On its turn the solutions for the field $\phi(x)$ are obtained by substituting the classical solutions of Eq. (16) into Eq. (13), namely

$$
\phi_{ \pm}^{A}(x)= \pm \frac{\sqrt{A}\left[A e^{\mp 4 \sqrt{A} r\left(x-x_{0}\right)}-\left(C^{2}-4 A B\right)\right]}{\left(\sqrt{A} e^{\mp 2 \sqrt{A} r\left(x-x_{0}\right)}+C\right)^{2}-4 A B} .
$$

The above solutions are valid if the parameters satisfy the conditions $A>0$ and $C^{2} \neq 4 A B$. The behavior of the above solutions are plotted in Fig. 1 for the parameters $r=$ 0.4 and $b=0.6$. One can observe that in both pairs of solutions, $\left(\phi_{+}, \chi_{+}\right)$and $\left(\phi_{-}, \chi_{-}\right)$, the field $\chi(x)$ exhibits a lumplike profile and the field $\phi(x)$ a kinklike profile. This behavior is also found in many systems of two interacting solitons reported in the literature.

More recently [11] it has been shown that many models of two interacting solitons, very similar to this one with explicit Lorentz symmetry breaking that we are presenting here, can also exhibit kinklike behavior for both of the soliton fields, depending on the range of the parameters of the model. Inspired by this achievement, we have shown that it is also possible to have kinklike profiles for both of the fields, for particular values of the parameters $r$ and $b$, in the model treated here. In fact if one takes $b=$ $2(r-1) / \sqrt{r}$, which corresponds to one of the solutions with $C^{2}=4 A B$, and $r>1 / 2$ in Eqs. (18) and (19), we obtain the following forms for the fields

$$
\chi_{ \pm}^{B}(x)=\frac{4(2 r-1)}{r\left(\sqrt{2 r-1} e^{\mp 2 \sqrt{2 r-1}\left(x-x_{0}\right)}+4 \sqrt{r}\right)}-\frac{r-1}{r \sqrt{r}}
$$

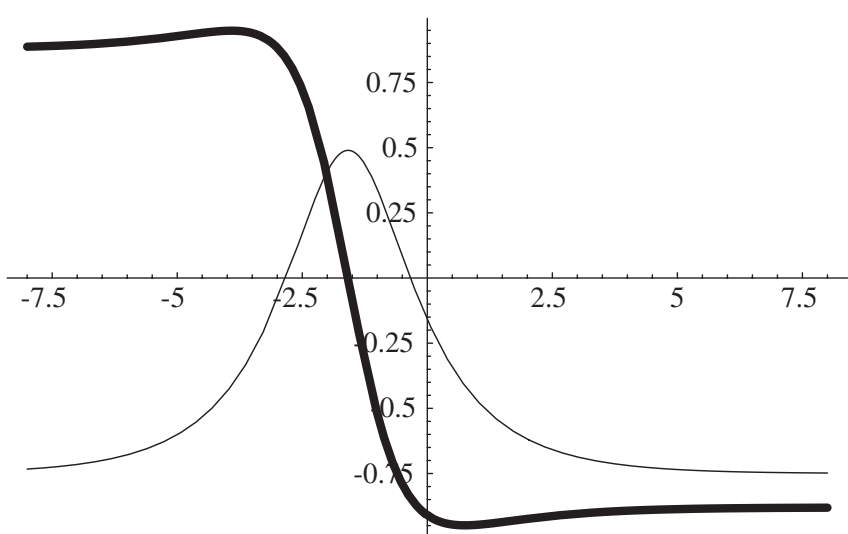

FIG. 1. Typical type- $A$ kink profile (for $r=0.6, b=0.4$ ). The thin line corresponds to the field $\chi_{+}(x)$ and the thick line to the field $\phi_{+}(x)$. Both were calculated for $c_{0}=0$. 


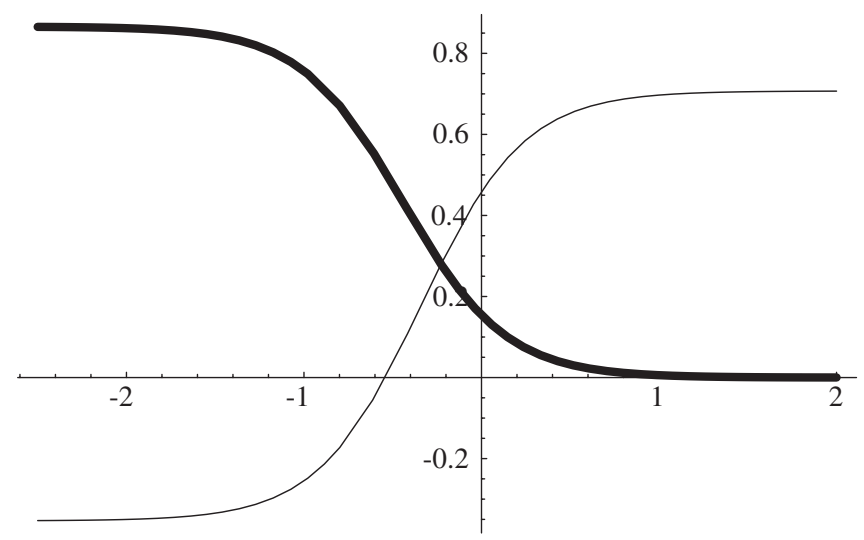

FIG. 2. Typical type- $B$ kink profile (for $r=2$ ). The thin line corresponds to the field $\chi_{+}(x)$ and the thick line to the field $\phi_{+}(x)$. Both were calculated for $c_{0}=0$.

and

$$
\phi_{ \pm}^{B}(x)= \pm \frac{(2 r-1)}{r\left(\sqrt{2 r-1}+4 \sqrt{r} e^{ \pm 2 \sqrt{2 r-1}\left(x-x_{0}\right)}\right)} .
$$

In Fig. 2 we present the behavior of the above kink solutions for $r=2$.

One could interpret these solutions as representing two kinds of torsion in a chain, represented through an orthogonal set of coordinates $\phi$ and $\chi$. So that, in the plane $(\phi, \chi)$, the type- $A$ kink corresponds to a complete torsion and the type- $B$ corresponds to a half torsion, similar to what has been done in [11]. In fact, we can observe that the degenerate vacua localized at $\left(0, \frac{1}{\sqrt{r}}\right) ;\left(0,-\frac{1}{\sqrt{r}}\right) ;\left(\sqrt{1-\frac{b^{2}}{4 r}},-\frac{b}{2 r}\right)$; $\left(-\sqrt{1-\frac{b^{2}}{4 r}},-\frac{b}{2 r}\right)$, are connected through both types of kinks. The type- $A$ kinks connect the last two vacua, which also can be connected through type- $B$ kinks in two steps, first from $\left(\sqrt{1-\frac{b^{2}}{4 r}},-\frac{b}{2 r}\right)$ to $\left(0, \frac{1}{\sqrt{r}}\right)$, and then to $\left(-\sqrt{1-\frac{b^{2}}{4 r}},-\frac{b}{2 r}\right)$. This explains the fact that the pairs of type- $B$ solutions have a BPS energy lower than that associated to the type- $A$ soliton solutions. This can be shown by substituting the asymptotic values of the solutions in Eq. (5), that is, for the type- $A$ solutions we find $E_{\mathrm{BPS}}^{A}=$ $\frac{4}{3} A \sqrt{A}$, and $E_{\mathrm{BPS}}^{B}=\frac{2}{3} A \sqrt{A}$ for the type- $B$ solutions. Really it should be expected that the energy spent to connect these vacua should be the same, in order to warrant the stability of both kinks.

\section{GENERALIZED MODELS}

In what follows, we will study a more general model contemplating a number of particular cases which have been studied in the literature, including the previous and some other new ones. For this, we begin by defining the superpotential

$$
W(\phi, \chi)=\frac{\mu}{2} \phi^{N} \chi^{2}+F(\phi),
$$

such that Eq. (7) is given by

$$
\frac{d \phi}{d \chi}=\frac{F_{\phi}+\frac{\mu}{2} N \phi^{(N-1)} \chi^{2}-g_{1}(\phi, \chi)}{\mu N \phi^{N} \chi-f_{1}(\phi, \chi)},
$$

where $F_{\phi}=d F / d \phi$. The space component of the functionals terms responsible for breaking the Lorentz symmetry explicitly, namely, $f_{1}(\phi, \chi)$ and $g_{1}(\phi, \chi)$, are to be chosen more general than those of the model discussed previously and conveniently such that the integration of Eq. (23) is possible. Based on the successful generalization of models of interacting solitons also carried out in Ref. [11] and in the development of the model of the previous section, a possible generalized model can be constructed by choosing

$$
F(\phi)=\frac{1}{2} \phi^{N}\left(\frac{\lambda}{N+2} \phi^{2}+\frac{\gamma}{N}\right),
$$

and the following forms for the functionals $f_{1}(\phi, \chi)$ and $g_{1}(\phi, \chi)$,

$$
f_{1}(\phi, \chi)=b \phi^{N} \chi, \quad g_{1}(\phi, \chi)=a \phi^{N-1} \chi,
$$

where $N$ is a positive integer number, $\lambda$ and $\gamma$ are constants, and the parameters $a$ and $b$ can be thought of as space components of two-vectors pointing out in some preferred direction in space-time and responsible for breaking the Lorentz symmetry.

The corresponding equation for the dependence of the field $\phi$ as a function of the field $\chi$ is now given by

$$
\frac{d \phi}{d \chi}=\frac{1}{2} \frac{\mu N \phi^{N-1} \chi^{2}+\phi^{N-1}\left(\lambda \phi^{2}+\gamma\right)-2 a \phi^{N-1} \chi}{\mu \phi^{N} \chi-b \phi^{N}} .
$$

Now, by performing the transformations

$$
\sigma=\frac{1}{2 \mu}\left(\lambda \phi^{2}+\frac{N b^{2}}{\mu}+\gamma-\frac{2 a b}{\mu}\right)
$$

and

$$
\varsigma=\mu \chi-b
$$

we get

$$
\frac{d \sigma}{d \varsigma}-\frac{\lambda \sigma}{\mu \varsigma}=\frac{N \lambda}{2 \mu^{3}} \varsigma+\frac{\lambda}{\mu^{3}}(N b-a) .
$$

The above equation is very similar to Eq. (12) and can be easily integrated out. Its general solution in the case $\lambda \neq \mu$ and $\lambda \neq 2 \mu$ is

$$
\sigma(\mathrm{s})=\frac{\lambda(N b-a)}{\mu^{2}(\mu-\lambda)} \mathrm{s}+\frac{N \lambda}{2 \mu^{2}(2 \mu-\lambda)} \mathrm{s}^{2}+c \mathrm{~s}^{\lambda / \mu},
$$

where $c$ is an arbitrary integration constant. The solutions 
for Eq. (29) in the cases $\lambda=\mu$ and $\lambda=2 \mu$ can also be obtained, but we will not deal with them here.

We substitute Eqs. (27), (28), and (30) in one of the Eqs. (4) to obtain the following first-order equation of motion for the field $s$

$$
\begin{aligned}
\frac{d \varsigma}{d x}= & \pm \mu^{1-N / 2} \varsigma\left[\frac{N}{2 \mu-\lambda} \varsigma^{2}+\frac{2(N b-a)}{\mu-b} \varsigma\right. \\
& \left.+\frac{c \mu}{\lambda} \varsigma^{\lambda / \mu}-N b^{2}-\gamma \mu+2 a b\right]^{N / 2} .
\end{aligned}
$$

This last equation can be solved analytically or numerically, depending on the values of the parameters. For the particular case with $N=2,2 b=a$, and $c=0$ we obtain very simple kink solutions for both of the fields $\phi(x)$ and $\chi(x)$, as can be verified from the behavior of the solution for the field $s(x)$

$$
\varsigma(x)= \pm \frac{\sqrt{B} e^{B x}}{\sqrt{1+A e^{2 B x}}},
$$

where $A=2 /(2 \mu-\lambda)>0$ and $B=\left(\gamma \mu-2 b^{2}\right) / \lambda>0$, and by substituting (32) in Eqs. (27), (28), and (30).

The construction of an even more general model which includes nonlinear dependence on the field $\chi(x)$ can be carried out by following the generalization proposed in Ref. [11]. This can be accomplished by choosing the following form of the superpotential

$$
W_{N M}(\phi, \chi)=\frac{\mu}{M} \phi^{N} \chi^{M}+F(\phi),
$$

where $F(\phi)$ is given by Eq. (24) and $M$ is a positive integer. In order to include the terms responsible for breaking the Lorentz symmetry and to obtain a solution for the differential equation (7) it is reasonable to choose the functionals $f_{1}(\phi, \chi)$ and $g_{1}(\phi, \chi)$ in the following forms

$$
f_{1}(\phi, \chi)=b \phi^{N} \chi^{M-1}, \quad g_{1}(\phi, \chi)=a \phi^{N-1} \chi^{M} .
$$

With this generalization Eq. (7) can be written in the form

$$
\frac{d \varphi}{d \chi}-\frac{\varphi}{(\mu-b) \lambda} \chi^{1-M}=\frac{(\mu N-2 a M)}{M(\mu-b) \lambda} \chi,
$$

where $\varphi=\lambda \phi^{2}+\gamma$.

Equation (35) is similar to the one which appears in Ref. [11]. It admits the solution

$$
\begin{aligned}
\varphi(\chi)= & \exp \left[-\frac{1}{\lambda(\mu-b)} \frac{1}{M-2} \chi^{(2-M)}\right] \\
& \times\left[\tilde{c}_{1}+\frac{2^{M /(M-2)}}{M(M-2)} \frac{\mu N-2 a M}{2 \lambda(\mu-b)} \chi^{2}\right. \\
& \times\left(\frac{\chi^{(2-M)}}{(M-2)}\right)^{2 /(M-2)} \\
& \left.\times \Gamma\left(\frac{2}{(M-2)}, \frac{1}{\lambda(\mu-b)} \frac{\chi^{(2-M)}}{(M-2)}\right)\right],
\end{aligned}
$$

where $\tilde{c}_{1}$ is an arbitrary integration constant and $\Gamma(a, z)=$ $\int_{z}^{\infty} t^{a-1} e^{-t} d t$ is the incomplete Gamma function.

\section{CONCLUSIONS}

We have been able to generalize a model presented recently in Ref. [10] which incorporates the phenomena of solitons interactions and the Lorentz symmetry breaking. The generalization has been carried out in two ways. We have found nontrivial classical solutions which exhibit kinklike behavior for both of the interacting fields and, consequently, with BPS energy lower than that associated with the usual solutions presented previously for the same model. Another interesting aspect of the kinklike solutions rests on the study of the stability of the solutions against small time-dependent linear perturbation. At least for some models with only one scalar field, it has been shown in Ref. [20] that models with kinklike solutions possess the stability of these solutions, on the other hand, models with lumplike classical solutions are unstable. For two interacting scalar fields the problem is cumbersome, even though the authors of Ref. [10] have been able to show, based on very elegant and general arguments, that the solutions found there, even with lumplike configurations for one of the fields, are stable. We understand that the analysis of the stability carried out in Ref. [10] is valid for reference frames in which $b_{0}=0$, where $b_{0}$ is the time component of the two-vector responsible for the Lorentz symmetry breaking. For reference frames in which $b_{0} \neq 0$ the analysis has not been done. Moreover, usually kinks arise in symmetry breaking models, which naturally do have degenerate vacua. In such cases there is a discrete symmetry transformation connecting those vacua, which in a certain way guarantees the quantum stability of the kinks. The discrete symmetry, $\phi, \chi \rightarrow-\phi,-\chi$, present when $b=0$ is no longer manifested in the model with Lorentz symmetry breaking here considered, what might indicate that the vacuum degeneracy is accidental, perhaps implying the instability against quantum corrections. It is remarkable, however, that the vacua of this model are present no matter what the particular values of the potential parameters are.

We have also proposed generalizations of the model of Ref. [10] by introducing nonlinear terms that break the Lorentz symmetry. This last generalization was possible thanks to the successful generalization carried out in Ref. [11] which deals with a Lorentz symmetric twodimensional model of interacting scalar fields.

\section{ACKNOWLEDGMENTS}

The authors A. S. D. and M. H. thank CNPq and FAB to FAPESP for the financial support. We also thank Professor D. Bazeia for introducing us to this matter and to Ref. [10]. This work has been finished during a visit of A. S. D. within the Associate Scheme of the Abdus Salam ICTP. 
[1] S. M. Carroll, G. B. Field, and R. Jackiw, Phys. Rev. D 41, 1231 (1990).

[2] D. Colladay and V. A. Kostelecky, Phys. Rev. D 55, 6760 (1997); 58, 116002 (1998).

[3] A. T. Azatov and J. L. Chkareuli, Phys. Rev. D 73, 065026 (2006).

[4] V. B. Bezerra, C. N. Ferreira, and J. A. Helayel-Neto, Phys. Rev. D 71, 044018 (2005).

[5] R. Lehnert and R. Potting, Phys. Rev. D 70, 125010 (2004).

[6] R. Bluhm, V. A. Kostelecky, and N. Russell, Phys. Rev. D 57, 3932 (1998).

[7] R. Rajaraman, Solitons and Instantons (North-Holland, Amsterdam, 1982).

[8] A. Vilenkin and E.P.S. Shellard, Cosmic Strings and Other Topological Defects (Cambridge University, Cambridge, England, 1994).

[9] D. Walgraef, Spatio-Temporal Pattern Formation (Springer-Verlag, Berlin, 1997).

[10] M. N. Barreto, D. Bazeia, and R. Menezes, Phys. Rev. D 73, 065015 (2006).
[11] A. de Souza Dutra, Phys. Lett. B 626, 249 (2005).

[12] M. K. Prasad and C. M. Sommerfield, Phys. Rev. Lett. 35, 760 (1975); E. B. Bolgomol'nyi, Sov. J. Nucl. Phys. 24, 449 (1976).

[13] L. J. Boya and J. Casahorran, Phys. Rev. A 39, 4298 (1989).

[14] D. Bazeia, M. J. dos Santos, and R. F. Ribeiro, Phys. Lett. A 208, 84 (1995).

[15] D. Bazeia and F. A. Brito, Phys. Rev. Lett. 84, 1094 (2000).

[16] D. Bazeia and F. A. Brito, Phys. Rev. D 61, 105019 (2000).

[17] D. Bazeia, R. F. Ribeiro, and M. M. Santos, Phys. Rev. E 54, 2943 (1996).

[18] D. Bazeia and E. Ventura, Chem. Phys. Lett. 303, 341 (1999).

[19] D. Bazeia, V. B. P. Leite, B. H. B. Lima, and F. Moraes, Chem. Phys. Lett. 340, 205 (2001).

[20] G. Flores-Hidalgo and N.F. Svaiter, Phys. Rev. D 66, 025031 (2002). 